\title{
Creatività ed etica della lettura di genere
}

\section{Anna Santoro}

Presidente dell'Associazione culturale Araba Felice

annasantoro1@libero.it

\begin{abstract}
Nell'intervento, a metà tra narrazione e riflessione critica, l'autrice, partendo dalle acquisizioni degli studi sulla «scrittura femminile» in Italia degli ultimi anni e dal proprio itinerario di ricerca, sottolinea il "grande scarto» compiuto dalle scrittrici sin dai secoli passati rispetto al Sistema Letterario canonizzato e, accogliendo le problematiche inerenti al rapporto lettura-testo, si ferma su alcune caratteristiche della lettura di genere quali il punto di vista, la relazione, lo sguardo, il posizionamento, il soggetto plurale, tutte necessariamente interne alla funzione creativa ed etica della buona lettrice.
\end{abstract}

Parole-chiave: soggettività, scrittura femminile, sistema letterario, canone.

\section{Abstract}

In her essay, half-way between narration and critical thinking, the author, moving from the knwoledge resulting from the studies on "female writing» conducted in Italy over the past years and from her own research, highlights how in the past centuries women writers started moving away from the official Literary System. Analysing the issues related to the reading-text relation, she investigates some pecularities typical of the gender reading, such as point of view, relationship, glance, positioning, plural subject, which are necessarily inherent in the creative and ethical function of the good woman reader.

Key words: subjectivity, feminine writing, literary system, canon.

Nella mentalità corrente, alla nozione di femminile e di maschile viene associata ancora la lettura che di essa ha fatto e fa la cultura patriarcale, ulteriore riprova di quanto sia in ritardo «'idea» di fronte al «fatto» che, da tempo, siamo in piena ridefinizione dei ruoli sessuali e delle caratteristiche tradizionalmente a essi legate. Ancora recentemente diverse studiose femministe si interrogano sulla nozione, oltre che di maschile e di femminile, di differenza, di genere, di sessualità, di corpo. ${ }^{1}$ Tra esse, Teresa De Lauretis sottolinea quanto sia necessa-

1. Cfr. Rosi BRAIDOTTI, «Oltre il genere-Analisi di una categoria controversa e, forse, non più utile», Leggendaria, settembre 2000 (con una scelta bibliografia sul tema); Paola BONO (a cura 
ria, per le donne, la «libertà di porre i termini di una questione, la libertà di non accettare definizioni». ${ }^{2}$

Anna Rossi Doria scrive: «La storia delle donne in Italia è riuscita a costruirsi in circa un quarto di secolo, una solida identità di disciplina, una sicura legittimazione e un buon avvio di istituzionalizzazione». ${ }^{3}$ Anna Rossi Doria si riferisce alle discipline storiche, ma il senso delle sue parole può essere applicato anche al fatto che esistono reti femminili internazionali di studi e di ricerche, dove si lavora allo sviluppo di dialogo tra diversi saperi e tra diverse modalità di approccio a testi e contesti, si lavora sulla nozione di percezione, di alterità, sulla soggettività discorsiva e sul soggetto nomade (Rosi Braidotti), sul soggetto eccentrico (Teresa De Lauretis), sullo sguardo e sul punto di vista, sulla (con)testualità. E si lavora, usando anche i risultati o i percorsi di ricerca «maschili», non solo senza traccia di competizione nei loro confronti, ma, con una, come dire?, scontata padronanza di se stesse che fa affrontare, con libertà (cioè con le problematicità che competono direttamente) le articolazioni, i dubbi e i problemi della ricerca stessa.

Penso all'interesse del volume Co(n)text: implicazioni testuali, a cura di Carla Locatelli (Trento 2000, ma il Convegno, del quale il volume raccoglie gli Atti, è del 1997), alle ricche discussioni e indagini portate avanti da riviste come Leggendaria, ai Convegni organizzati dalla Società delle Letterate, a contributi interessanti come Identità di genere e immagine femminile (Irrsae Puglia, 2000), a cura di Maria Vinella, o a Incroci di genere (Bergamo, University Press, 1999) a cura di Mario Corona, penso all'attenzione che negli ultimi è stata data alla ricerca di scrittrici italiane dei secoli passati, a piccole case editrici che rieditano testi altrimenti introvabili, alle Università che organizzano Masters post-universitari per le Pari Opportunità, ai Women' Studies che vanno prendendo piede, a molti lavori, tutti interessanti, seppure, o proprio per questo, diversi, di tante studiose che, in diversi ambiti disciplinari, portano contributi originali e utili per tutte/tutti. ${ }^{4}$

Eppure, in Italia ancora, in molti ambiti, espressioni del tipo «scrittura femminile» vengono usate in modo riduttivo e nello stesso tempo troppo generico, ancorate al tempo in cui si discuteva del femminile come "specificità". Oppure si cerca di fissare regole di scrittura, canoni, o addirittura il Canone

di), Questioni di teoria femminista, Milano: La Tartaruga, 1993, soprattutto il saggio di Elisabeth Grosz, "Firmare con il sesso: il femminismo dopo la morte dell'autore».

2. Cfr. Teresa De lauretis, Sui generi, Milano: Feltrinelli, 1996, p. 17.

3. Cfr. Anna Rossi DORIA, "Quale cultura per quale politica», Leggendaria, n. 23, settembre 2000, p. 10.

4. Cfr. per esempio, tre tipologie di ricerca molto differenti l'una dall'altra: Marta SEGARRA, Helena GOnZales Fernandez, Francesco Ardolino (a cura di), Rías de tinta. Literatura de mujeres en francés, gallego e italiano, Barcelona: Universitat de Barcelona, 1999; Adriana Chemello e Luisa RiCAldone, Geografie e genealogie letterarie... tra Settecento e Ottocento, Padova: Il Poligrafo, 2000; Franca Ferraris Cornaglia, Mirella Melis Zucca, Marcella Mocci Serri, Maria Luisa Viola, Donne, Due secoli di scrittura in Sardegna (1775-1950). Repertorio bibliografico, Cagliari: CUEC, 2001. 
della scrittura femminile, come se non fosse stata messa in discussione la stessa nozione di Canone, a qualsiasi genere lo riferiamo. Ben altro significato assume l'espressione sopra citata in contesti non italiani: si pensi alla écriture féminine di Hélèn Cixous, che è, come sottolinea Carla Locatelli, «un condensato della problematica del rapporto corpo-scrittura [...], espressione di una formidabile sintesi semantico-culturale». E più avanti: «[...] écriture féminine non indica un concetto, un'idea fissa; non è l'etichetta di un pensiero, né conferisce una patente di appartenenza a chi scrive». 5

Per quel che mi riguarda, ho sempre cercato di (imparare a) continuamente assumere e (ri)leggere i segni e i comportamenti delle donne come esempi (non modelli) dei possibili modi del femminile, non per una sorta di «prudenza scientifica», ma perché immagino e mi auguro che, a fronte del Sistema letterario tradizionale maschile, non si voglia contrapporre un analogo Sistema letterario femminile:

[...] il punto non è affermare l'esistenza o meno di un sistema letterario femminile che, fondandosi anch'esso su un automodello, elabori un sistema di siste$m i$ tale da poter essere contrapposto a quello maschile, e forse non è più (non è solo più) intaccare miti e stereotipi, svelandoli «di parte maschile», ma il punto è accogliere e fare emergere i soggetti femminili così come essi si sono mossi e mostrati di fatto. Ed essi, nelle opere dove la soggettività si manifesta tramite il linguaggio, si presentano estremamente complessi da leggere, perché ricchi di sfaccettature che non siamo abituate a valorizzare. ${ }^{6}$

La poesia nasce nel momento in cui si dà forma al desiderio, e non ha, in una ipotetica scala di valori, genere o nazionalità, età, ma... Ma il desiderio e lo sguardo sul mondo, la necessità poetica (Rilke, Lettere ad un giovane poeta), il progetto poetico (Gottfried Benn), sono diversi a secondo della vita, vissuta e guardata, di chi scrive. Virginia Woolf sottolinea come il romanzo «non viene fuori dall'immaginazione, non viene fuori all'improvviso [...]. La narrativa è come una tela di ragno che se ne sta attaccata in maniera forse lievissima alla vita. [...] quelle ragnatele non sono tessute a mezz'aria da creature incorporee, ma sono opere di esseri umani che soffrono, e sono strettamente legate a fatti grossolanamente materiali come la salute, il danaro e le case in cui abitiamo».

Per questo ritengo che l'indispensabile lavoro di recupero della produzione femminile italiana dei secoli passati serva non per contrapporla a quella maschile, né per un'ulteriore riprova di una non felice condizione femminile, né per recuperare miracolosamente capolavori perduti e con essi fissare un nuovo Canone, ma per visualizzare e accogliere linguaggi tutti da investigare, forti però delle riflessioni fatte in questi anni, non solo riguardo la differenza

5. Cfr. Carla Locatelli, «Questo lavoro d'analisi e illuminazione», in Paola Bono (a cura di), Scritture del corpo. Hélène Cixous, variazioni sul tema, Roma: Sossella, 2000, p. 22-23.

6. Anna SANTORO, Il Novecento. Antologia di scrittrici italiane del primo ventennio, Roma: Bulzoni, 1997, pag. 23.

7. Virginia Woolf, Una stanza tutta per sé, Torino: Einaudi, 1995, p. 85. 
di genere, ma anche riguardo il testo, i contesti, la lettura, il rapporto tra chi legge $\mathrm{e}$ ciò che viene letto.

Se assumiamo le riflessioni sul testo di Barthes, di Derrida, (che non sono in contraddizione come risulta chiaro nel lavoro della Locatelli, $\mathrm{Co}(n)$ testi, che apre il già citato volume di Trento), di Benveniste, la nozione di soggetto plurale della Harendt, ma anche considerazioni di Benjamin o di Foucault, e se allarghiamo il nostro sguardo accogliendo le problematiche relazioni tra politica e filosofia, così come vengono trattate da Jean-Luc Nancy in Essere singolare plurale, ${ }^{8}$ non possiamo non convenire che «la comprensione non coincide con il significato di un testo", ${ }^{9}$ e che il punto è, nei confronti del testo scritto, ma anche nei confronti del testo mondo, che leggere è sempre «un'avventura dello sguardo». ${ }^{10}$

La ricerca e l'analisi delle scritture delle donne in Italia, che si sono sviluppate negli ultimi venticinque anni, grazie alle letture dei testi, al lavoro di storiche e studiose di varie discipline, alla ricostruzione dei contesti e di scenari, alle riflessioni della critica femminista, all'assunzione della differenza (della coscienza e del posizionamento di genere) da parte di chi legge (oltre che da parte di chi scrive), hanno posto il problema della rivisitazione della tradizionale nozione di Sistema letterario e di Letteratura italiana come corpus di scritture neutre, in un momento in cui (la coincidenza non è casuale) era avvertita anche da parte di alcuni studiosi l'esigenza di affrontare la nozione di «Storia Letteraria» a suo tempo codificata da De Sanctis (La Letteratura Italiana di Einaudi, curata e ideata da Asor Rosa, ne è segno tangibile), e prima ancora che venisse importato il "problema del Canone» dalle Accademie anglosasso$\mathrm{ni}{ }^{11}$ (con finalità e modalità molto differenti).

Eppure, quando cominciammo ad approfondire il lavoro sulla produzione femminile, ci trovammo di fronte al problema di cosa fosse la lettura, di cosa fosse un testo (inteso anche come scenario letterario, sociale, politico: contesto dei testi). Scambiandoci doni con gli autori che ho sopra nominato (quelli che si interrogavano appunto sulla consistenza del testo) e riflettendo sulle nostre pratiche e sui nostri saperi, elaborammo, e continuiamo ad elaborare, una nozione di lettura che è accoglienza ed elaborazione, scambio, tramite di conoscenza dell'altra/altro e di sé. La lettura è il risultato della relazione tra occhio che guarda e cosa guardata (il testo, ma anche il testo-mondo), e, come è difficile de-finire in contorni netti e stabili il testo (e i contesti), così lo è anche definire chi è a leggere. Qui è il punto.

8. Cfr. Jean-Luc NANCY, Essere singolare plurale, Torino: Einaudi, 2001.

9. Carla LOCATELlI, "Co(n)testi», in Co(n)text: implicazioni testuali, a cura di Carla Locatelli, Trento: Università degli Studi, 2000, p. 26.

10. EAD., p. 35. Sulla «lettura», cfr. l'interessante saggio di Vita FORTUNATI, «Il lettore sublime», in Atti del Convegno: il sublime-creazione e catastrofe della poesia, Bologna: Nuova Serie, 1-2, 1984, p. 77-92; notizie e riflessioni utili (ma di altro genere) anche in Albert MANGUEL, Una storia della lettura, Milano: Mondadori, 1997.

11. Cfr. recentemente: Harold Bloom, Il Canone occidentale, Milano: Bompiani, 1999. 
Non basta più pensare ad un imprecisato punto di vista femminile per ritenere che esso sia in grado di intrecciare relazione con i testi femminili. Certo, c'è un corpo nelle scritture delle donne e c'è un corpo della lettrice, c'è una scelta di posizionamento sia in chi scrive sia in chi legge. Ma c'è anche altro. Così, credo sia utile fermarsi su una questione centrale, punto di partenza per qualsiasi discorso che si interroghi sulle scritture delle donne, sul femminile in lettura, sulla differenza di genere, sulla ricostruzione dei contesti, e così via, e cioè vorrei porre in primo piano il soggetto e i modi di questo interrogarsi, cioè la figura della lettrice.

Prima, però, apro una parentesi per dare ragione del tragitto che io stessa ho attraversato (mi si perdonino dunque le autocitazioni, funzionali al ragionamento che sto impostando): questa metodologia di ricerca appartiene a una delle forme possibili del femminile, e qualifica, a mio avviso, la ricerca di genere come creativa, ${ }^{12}$ (non strettamente "scientifica» nell'accezione data comunemente a questo termine), nel senso di eversiva, fuori dai canoni, dettata dalla necessità di ricerca (e per questo a suo modo scientifica). E dunque anche etica.

Ancora prima delle modalità, sono importanti le motivazioni, strettamente intrecciate, che spinsero me (e tante altre), ${ }^{13}$ alla fine degli anni ' 70 a studiare le scrittrici italiane, e cioè l'interesse per la scrittura, per la poesia, per la letteratura, e la partecipazione appassionata agli eventi degli anni ' 70 , in particolare al femminismo. A quel tempo, gli studi sulle donne, sulla scrittura femminile, sulla creatività delle donne, avevano tutti come punto di riferimento le letterature straniere. In Inghilterra sì, c'erano state scrittrici, in Francia, in America, ma in Italia, si affermava, niente. Questa presunta assenza di produzione femminile italiana, accettata e spiegata con varie motivazioni da studiosi (che, se la prendevano in esame, la davano per scontata e/o la ribadi-

12. Se le lettrici, le studiose, non fossero (state) creative, non avremmo mai ri-cominciato a leggere la produzione femminile italiana. E a interrogarci su di essa.

13. Tra la fine degli anni '70 e i primi anni degli anni ' 80 , ricordo alcuni lavori di studiose che per me furono importanti: Franca BASAGLIA Ongaro, Una voce, Milano: Il Saggiatore, 1982; Angela BianCHInI, Voce donna, Milano: Bompiani, 1979; Ginevra BOMPIANI, Lo spazio narrante, Milano: La Tartaruga, 1978; Bruna CONTI e Alba MORINO, Sibilla Aleramo e il suo tempo, Milano: Feltrinelli, 1981; Patrizia MAGLI (a cura di), La donna e i segni: scrittura, linguaggio, identità nel segno della differenza femminile, Ancona: Il lavoro editoriale, 1985; Giuliana Morandini, La voce che è in lei, Milano: Bompiani, 1980; Luisa Muraro, La Signora del gioco, Milano: Feltrinelli, 1976; Anna Nozzoli, Tabù e coscienza femminile nella letteratura italiana del Novecento, Firenze: La nuova Italia, 1978; Ginevra CONTI Odorisio, Donna e società nel Seicento, Roma: Bulzoni, 1979; Franca PIERONI BorTOLOTTI, All'origine del movimento femminile in Italia, Torino: Einaudi, 1975; Marina ZANCAN, Nel cerchio della luna, Venezia, 1983; EAD. "La donna», in A. ASOR RosA (a cura di), Letteratura italiana. Le questioni. V, Torino: Einaudi, 1986. Ma influirono su me e sul mio lavoro anche molte studiose (di altre discipline o di altre letterature) pioniere di quegli anni (e che hanno poi continuato), come Gisela BocK, Rosi BraidotTI, Annarita BuTTAFUOCO, Lea Melandri, Biancamaria Frabotta, Nadia Fusini, Ellen Moers, Adrienne Rich, Anna ROSSI DORIA, Elaine SHOWALTER, e, sopra tutte, Virginia WOOLF che naturalmente non appartiene a quegli anni ma resta fondamentale, in questo caso come saggista, per chiunque si interroghi sulle scritture femminili. 
vano) e anche da studiose (che l'attribuivano alle difficili condizioni delle donne in Italia), era in realtà un luogo comune, funzionale a discorsi altri, e non un fatto verificato. Per cercare risposte, sia sul piano letterario sia su quello, diciamo, «ideologico», avvertivo l'urgenza di affrontare il problema avviando prioritariamente una ricerca sistematica di cosa avessero scritto in Italia le donne.

È importante ricordare a questo proposito che, mentre la critica ufficiale (maschile), agli inizi degli anni '80, consacrava la «morte dell'autore» (ma il discorso è tuttora aperto), l'attenzione (mia e di altre), pur partendo dal testo e grazie alla lettura dei testi, ricercava nel testo l'autrice, per articolare la Storia del soggetto femminile. Sottolineo anche che nel pensare una ricerca a tappeto della produzione femminile italiana non cercavo scrittrici che mostrassero coscienza di genere, identità, assunzione della differenza sessuale: desideravo vedere tutti i testi e successivamente interrogarmi sulle modalità di lettura. ${ }^{14}$

La mia ipotesi di lavoro, dunque, era chiara, sia pure visionaria (creativa): ero convinta che ce ne fossero state, di scrittrici, ero convinta che fosse necessario recuperarne memoria, ero convinta che servisse alle donne sapere di possedere una tradizione. («La produzione letteraria femminile in Italia è pressoché sconosciuta perché inesplorata [...]»). ${ }^{15} \mathrm{Ed}$ ero convinta che, poiché è nel linguaggio che si dà forma alla visione del mondo, nel linguaggio (in questo caso "di parola») dovesse/potesse essere studiata la differenza di genere.

Nel frattempo, il mondo cambiava, e cambiava il rapporto tra soggetto e oggetto. Cambiava la nozione di cultura (maschile e occidentale), non più autoreferente.

Una volta era il verbo: c'era il testo e c'era il lettore. Si raccontava che la scrittura fosse neutra, e che la lettura fosse neutra. In realtà si riteneva che la scrittura maschile (le scritture maschili) fossero il generale, il centrale, l'unico soggetto possibile, l'unico punto di vista idoneo a stabilire canoni e scale di valori, senza che fossero denunciati/e di parte. Questo soggetto maschile universale (al suo interno, certo, articolato) nutriva la nozione di libro (di mondo) come oggetto chiuso, magari segreto, da penetrare per coglierne i significati: in questo modo poteva essere detta neutra sia la scrittura sia la lettura.

Grazie al Catalogo della scrittura femminile italiana presente nei fondi della Biblioteca Nazionale di Napoli (dalle origini della stampa al 1860), ${ }^{16}$ verificai

14. Evidentemente c'è una parte di me che sente il bisogno di fare elenchi, registrare, acquisire dati, intrecciare percorsi, se ho da poco messo in Rete il Progetto Dominae, Dizionario biobibliografico delle donne, sul Sito della mia Associazione: www.arabafelice.com.

15. Cfr. Anna SAntoro, "Gli amori di una letterata” della Signora D», Esperienze Letterarie, 1980, n. 2.

16. Catalogo della scrittura femminile italiana presente nei fondi della Biblioteca Nazionale di Napoli (dalle origini della stampa al 1860), Napoli: Centro Studi condizione della donna. Comune di Napoli, 1984. Il Catalogo, realizzato con un piccolissimo contributo del CNR (tramite fu il carissimo amico Giancarlo Mazzacurati, della cui perdita la cultura italiana non si consolerà mai, e neanche io), fu consegnato all'Università di Napoli nel 1979. La successiva edizione del Catalogo (Napoli: Dick Peerson, 1990), rivista e rielaborata, arriva al 1900 ed è accompagnata da una Guida al Catalogo, dove sono raccolti interventi di studiose di varie discipline. 
che davvero tante donne avevano scritto e pubblicato in Italia. Nel leggerle, capii quanto fosse importante la figura della lettrice, e capii (accolsi) che per leggere quei testi non poteva essere usata la griglia consueta, la consueta scala di valori:

Questa inchiesta sulla produzione femminile italiana a stampa [...] sta a significare una messa in discussione di analisi non solo di parte, ma vecchie [...]. Il lavoro nasce per conoscere ciò che hanno scritto le donne in Italia. [...] non si conosce questa produzione che dunque non viene usata nelle analisi" (p. 3). [...] «Solo un lavoro sistematico permette oggi di riscoprire testi condannati all'oblio da un sistema di valori che non è più quello moderno" (p. 4). [...] «...il metro di analisi di queste opere è altro da quello ufficiale [...] Questo lavoro di fatto serve a mostrare la base culturale, grazie alla quale o nonostante la quale, altre donne $[\ldots]$ hanno saputo e voluto scegliere di esprimere se stesse e il loro rapporto con la realtà (p. 5-6). ${ }^{17}$

Grazie al Catalogo, che, con la sua ricchezza, mi ha permesso di partire costantemente dai testi e dunque di basare sulla lettura la riflessione critica, andavo scoprendo decine e decine di nomi poco o per niente conosciuti, che leggevo, certo, perché opere di donne - era questa la mia ricerca - ma non per «indagare il femminile». In certo senso cercavo di dimenticare che fosse "scrittura femminile», ${ }^{18}$ cercavo di assumere uno sguardo, di parte e non neutro certo, ma quanto più vergine possibile, attento, e nello stesso tempo di abbandonarmi al piacere della lettura. Che era evidente perché, pur essendo lettrice "colta e smaliziata», provavo per la prima volta il piacere della complicità, della comunanza, ${ }^{19}$ dell'identificazione, ma soprattutto scoprivo cose nuove.

A proposito dell'identificazione e delle comunanze: esse immediatamente procurano felicità, ma a volte fastidio. La lettrice, è vero, ritrova nel testo femminile elementi che le appartengono: dai più semplici pensieri o visioni vissute da bambina, alle curiosità sperimentate, allo spazio narrativo, al ritmo, e poi alla segnalazione di sentimenti, di oggetti di curiosità, di condivisione, di rapporti... Ma accade anche che questa felicità si tramuti a tratti nel suo contrario, cioè in una sensazione quasi fastidiosa. A rifletterci sono giunta a ritenere che, per una donna, leggere una donna è trovarsi a contatto con qualcosa di troppo conosciuto, scontato, semplice. E certe volte questo non dà limpidezza

\section{Cfr. Anna Santoro, Prefazione al Catalogo..., cit., (Napoli, 1984).}

18. Virginia WOOLF raccomanda alle scrittrici di non essere distratte, nello scrivere, da risentimenti e rivendicazioni: in certo senso, precisa, bisogna dimenticare di scrivere da donne e farlo semplicemente. Aggiunge che però anche gli uomini raramente hanno dimenticato di essere uomini e spesso anche la loro è scrittura "risentita».

19. Cfr. Robert ESCARPIT, Sociologia della letteratura, Napoli: Guida, 1977. In particolare (p. 97 e seg.) si riferisce alle "comunanze» (di cultura, di evidenze, di linguaggio) che garantiscono il passaggio della comunicazione tra chi scrive e il suo pubblico. Cfr. anche: Anna SANTORO, "Narrativa di fine Ottocento. Le scrittrici e il pubblico", Italiana IV, 1992, p. 103-126. 
di sguardo. Perché è una novità. Perché abbiamo sempre letto cercando di identificarci in (di accogliere) qualcuno di completamente diverso da noi, l'eroe, il protagonista, allenandoci ad assumere lo sguardo, il punto di vista, l'oggetto d'attenzione che lo scrittore ci presentava. Le scritture delle donne, imparai, se vengono segnate come categoria compatta e data una volta per tutte, corrono il rischio di essere lette in modo errato proprio dalle donne.

Sono le cose nuove, le nuove soluzioni narrative, le nuove metafore, i nuovi oggetti di attenzione che sono importanti. Potrei fare tanti esempi, ma tralasciando casi come la sorpresa alla lettura dei testi poetici ritrovati nelle Raccolta della Bergalli ${ }^{20} \mathrm{o}$ in quella di Bulifon, ${ }^{21}$ che mi autorizzò a interrogarmi sulla tradizionale lettura della presenza e del ruolo delle donne nelle corti del ' $500,{ }^{22}$ o il divertimento a leggere una serie di libretti che non posso che definire "curiosi», o la noia di fronte agli «scritti d'occasione», che restano però di grande interesse sul piano del costume, o ancora le riflessioni che mi destarono opere di storiche e di pensatrici, rimando ai miei lavori e accenno velocemente alla meraviglia, che si andò trasformando sempre più in ammirazione, che mi procurò trovare un numero tanto alto di scrittrici, e una qualità così certa, tra l'ultimo ventennio dell'800 e il primo del $900 .^{23}$

Caterina Percoto, Rosalia Piatti, Luisa Saredo, Maria Savi Lopez, Matilde Gioli, Elda Gianelli, Grazia Mancini Pierantoni, Anna Zuccari Radius (Neera), Maria Antonietta Torriani (Marchesa Colombi), Evelina Cattermole Mancini (Contessa Lara), e molte altre, grazie all'introduzione di tematiche nuove (la guerra, le trasformazioni economiche sociali delle donne nella metà-

20. Cfr. Luisa Bergalli GozZI, Componimenti poetici delle più illustri rimatrici di ogni secolo.., Venezia: Mora, 1726.

21. Cfr. Rime di cinquanta illustri poetesse..., Napoli: Bulifon, 1695.

22. Cfr. Anna SANTORO, «Ricerca e lettura delle scritture delle donne in Italia. 1) Questioni di metodo", Esperienze Letterarie, n.3, 1990; EAD., "Per un'analisi dello stato socio-economico delle scrittrici italiane (dalle origini della stampa al 1860): appunti su produzione femminile, stampa, mercato", Prospettive Settanta, n. 1, 1984, (poi, rivisto, in Guida al Catalogo, cit.); EAD., (a cura e con Prefazione), I secreti de la Signora Isabella Cortese, Venezia: Bariletto, 1561. Stampa anastatica, Napoli, 1999.

23. Per le notazioni, e per le scrittrici nominate, rimando ai miei lavori, specialmente: Narratrici italiane dell'800 (Napoli: Federico, 1987); «Il fatto è che ingrasso. Lettura di Un matrimonio in provincia della Marchesa Colombi», in Nunziante Cesaro A.-Marino S. (a cura di), Soggetto femminile e scienze umane, Bologna: Clueb, 1993; "Pagine di biblioteca», Leggendaria, Luglio-Agosto, 1993; "Intellettuali sulla scena», Leggendaria, Novembre-Dicembe, 1994; "Intellettuali del'800: operazione svelamento", Leggendaria, Marzo-Maggio, 1996; Il Novecento, Antologia di scrittrici italiane..., cit.; "Scrittrici», in Napoli e la Campania del Novecento, a cura dell'Istituto Croce e dell'Università di Napoli (in corso di stampa: per questo motivo non potrò segnalare le pagine a seguito di eventuali citazioni); Piccola Antologia di scrittrici campane, Napoli: Intramoenia, 2001; "Leggere, scrivere, linguaggi del corpo", in Leggendaria, 2001, n. 27-28; «Leggere le scrittrici (italiane) del passato: questione di metodi. Impressioni e ricordi (1856-1864), Diario di Grazia Mancini Pierantoni», in Atti del Congresso internazionale "Rappresentare-rappresentarsi: firmato donna.», Siviglia 24-28 Ottobre 2001 (in uscita: anche per questo testo le eventuali citazioni non porteranno numero di pagina). 
fine dell'800, il matrimonio di convenienza), o grazie al capovolgimento geniale di favole e di luoghi comuni, o alla prospettiva diversa dalla quale guardavano e raccontavano le cose, mi fecero prendere atto definitivamente dell'ormai noto punto di vista femminile. («Fisionomie molto diverse l'una dall'altra, queste scrittici [...] introducono nella narrativa italiana una grossa novità, e cioè che la voce narrante è quella di donna. Semplicemente. Portano esse la loro scrittura come portano il volto, il corpo, che è corpo e volto di donna $[. .$.$] ". E poco dopo: "[...] centro del loro scrivere è [...] scrivere$ dal punto di vista di donna». ${ }^{24}$ Punto di vista che ritrovai in Fanny Salazar, Aurelia Folliero, Sibilla Aleramo, Annie Vivanti, Carola Prosperi, Eugenia Codronchi (Sfinge), Anna Franchi, Paola Drigo, Clelia Pellicano, Lina Pietravalle, Maria Messina, e in tutte le altre che ho avuto l'avventura di conoscere.

Non sto affermando che sono uguali, queste scrittrici, ma che hanno in comune molte cose: il punto di vista non è solo (!) un posizionamento diverso di fronte al mondo, è vedere un altro mondo.

Ciò che ancora si stenta a comprendere è che lo spazio creativo delle scrittrici é diverso (da quello degli scrittori): la percezione della realtà, delle scoperte scientifiche, degli avvenimenti pubblici, dei fatti culturali, dei dibattiti politici o intellettuali, e dunque la tensione a (e le modalità per) «dare forma» alla propria percezione [...], per le donne è diversa. Le cose stesse, scelte per la propria attenzione $[\ldots]$ indicano come, alla percezione femminile della realtà, quelle e non altre risultino degne di nota: sono quelle le esperienze memorabili che vanno raccontate. Dunque, [...] le donne hanno vissuto e recepito a loro modo (e, all'interno di questo, ciascuna a suo modo) gli eventi pubblici che nel frattempo facevano i pensieri, le convinzioni, le idee e la lingua degli scrittori. ${ }^{25}$

Molte di queste scrittrici fondarono giornali (che diventavano, come racconta la Salazar, non solo salotti intellettuali e politici, ma centri di riferimento per le donne «comuni») e/o collaborarono alle più importanti testate del tempo (La Nuova Antologia, Lillustrazione Italiana...), alcune furono attiviste o perlomeno legate ai movimenti femminili internazionali, inviavano all'estero loro contributi, partecipavano a Convegni, giravano facendo conferenze, avviavano battaglie importanti. ${ }^{26}$ Ebbero tutte in comune, sia pure ciascuna a suo modo, il desiderio di vincere le paure e di mostrarsi. Ebbero in comune il fatto di essere «impegnate». Perché tutte furono portatrici di trasgressione (nel senso di «andare oltre»): ruppero i canoni, nella vita e nella scrittura, attaccarono i luoghi comuni in modo più o meno vistoso, si mostrarono in pubblico, vis-

24. Cfr. Anna Santoro, Narratrici italiane..., cit., p. 10-11 e p. 13-14.

25. Cfr. EAD., "Scrittrici», cit., passim.

26. Le citazioni e i riferimenti sono tantissimi e non posso non rinviare, per riscontri puntuali, ai miei lavori. Notizie utilissime anche nei lavori di Franca PIERONI BORTOLOTTI. Cfr. anche Michela De GiorgIO, Le italiane dall'Unità a oggi, Bari: Laterza, 1992. 
sero vite coerenti con il loro scrivere. Nei loro scritti, tutte riesaminano il concetto di «virtù», di «femminile», di forza e di debolezza, e riesaminano i luoghi comuni attorno al matrimonio, all'età, allo "zitellaggio", ma anche affrontano la trasformazione economico-sociale dell'epoca, riuscendo a trovare, sia le scrittrici provenienti da classi non privilegiate, sia le aristocratiche, nell'autonomia economica, una delle strade necessarie alla libertà propria e di tutte le donne. E tutte affrontano questioni generali come la guerra, il lavoro, la disoccupazione, i problemi del proprio territorio, in un modo che nessuno scrittore aveva ancora fatto.

Tutte scrivono di violenza quotidiana sulle donne, ciascuna sottolineando aspetti diversi, ma ritrovandosi a sottolinearne i principali. Grazia Mancini, Fanny Salazar, Neera, la Marchesa Colombi, l'Aleramo, e poi Clelia Pellicano, Anna Franchi, Lina Pietravalle, Maria Messina, Paola Drigo, Annie Vivanti, tutte, nel raccontare storie di violenza sulle donne, mettono in scena la cronaca quotidiana, il vissuto quotidiano delle donne, riuscendo a comunicare emozione, e soprattutto sono capaci di rappresentare la violenza come insita nella società patriarcale. ${ }^{27}$ Tutte danno vita ad uno scenario dove ad essere centrale sono le donne. Nei loro romanzi sfilano figure di donne, che si accampano protagoniste sulla scena, anche se ferite, deluse, ma mai risentite, sempre forti, a volte addirittura maternamente indulgenti e ironiche nei confronti degli uomini. ${ }^{28}$

La vita quotidiana femminile, lontana dall'essere unicamente cucina, casa, cicaleccio tra comari, paziente sopportazione, rivalità tra belle donne, scenario di folla femminile un po' anonima posta di sfondo al protagonista maschile centrale, com'è rappresentata nella letteratura maschile di quegli anni, diviene, allo sguardo delle scrittrici, oggetto di osservazione privilegiato per quelle che sono le "priorità» del vivere: così, la guerra è colta nel quotidiano femminile che subisce il dolore delle perdite e non riesce più a credere alle favole dell'onore (Percoto), il matrimonio è nel quotidiano fatto di convenienza o di violenza sessuale e di delusione sentimentale ed erotica (Saredo, Neera, Marchesa Colombi, Aleramo, Prosperi, Franchi, Codronchi, Serao...), la solidarietà maschile è volgare ammiccamento (De Donato, Pietravalle), l'amore è scelta di passione (Codronchi, Prosperi, Pietravalle, ma già prima Neera, Serao), nutrito anche di valenze nuove (la complicità, le condivisione di gusti e ideali), il sentimento della maternità, tanto decantato e strumentalizzato dalla let-

27. Il bellissimo Maria Zef, di Paola Drigo (1936) è esemplare.

28. È interessante notare che la moderna letteratura femminile italiana, quando l'autrice torna con la memoria alla storia passata, familiare o no, presenta spesso il mondo femminile centrale nell'esperienza pubblica e privata. Penso in particolare a Althénopis (Torino: Einaudi, 1981) di Fabrizia Ramondino, a Francesca e Nunziata (Anabasi, 1995, poi Cava dei Tirreni: Avagliano, 1998) di Maria Natale OrSINI, a Le viceregine di Napoli (Firenze: Giunti, 1997) di Luciana VIVIani, a Passioni di famiglia (Milano: Feltrinelli 1994) di Cristina CoMENCINI, a Posillipo (Rizzoli, 1997) di Elisabetta Rasy, a Le amiche di Carla (Napoli: Filema 1999) a firma di chi scrive questa nota. Cfr. A. SANTORO, "Scrittrici», cit.; EAD., "Leggere e scrivere, linguaggi del corpo", cit., passim. 
teratura ufficiale (maschile), viene rivisitato e a volte, sia pure dolorosamente, superato dall'amore di sé (Franchi, Aleramo).

Il punto è che le scrittrici svelano, per esempio, la violenza della condizione femminile, non solo perché interessa a loro e alle loro lettrici, ma prima ancora perché la vedono (anche la buona lettrice la vede e la vive e per questo valuta l'operazione culturale compiuta dalla scrittrice); svelano la brutalità e la profonda inumanità della guerra perché la soffrono e la vivono come scelta esclusivamente maschile, praticata sia per interessi materiali sia per l'incapacità di accettare differenze alla pari.

Il peso dello sguardo dell'altro sul comportamento femminile, sulla sua coscienza, sulla sua autonomia, è svelato da tutte le scrittrici che operarono in Italia tra la fine dell'Ottocento e i primi del '900. Per esempio dalla Marchesa Colombi, con la sua nitidissima analisi dell'amore, in "Un matrimonio in provincia», da Fanny Salazar, da Grazia Mancini, da Matilde Serao, da Lina Pietravalle, da Eugenia Codonchi, da Maria Messina, e da tante altre, che ammoniscono le donne a non guardarsi con lo sguardo dell'altro.

È questo il grande «scarto» compiuto dalla scrittura femminile: il mondo e i suoi valori, le abitudini, i comportamenti, le mentalità, vengono ribaltate. Lo spostamento del punto di vista comporta una visione del tutto inedita per l'esperienza letteraria. È una scelta di posizionamento ancora più ricca di conseguenze di quella, a quel tempo tentata da tanti scrittori, di guardare il mondo da parte del "popolo». Perché mentre gli scrittori (in modo diverso: Valera, Verga, Manzoni, altri) cercano, e prevalentemente per fini e con motivazioni «letterarie», di «mettersi dalla parte di», le donne sono "la parte» di cui trattano. Danno voce a se stesse. E incontrano la voce e lo sguardo delle loro lettri$\mathrm{ci}^{29}$ del loro pubblico:

Anche il pubblico, femminile e maschile, è nello sguardo delle scrittrici e fa parte dello scenario: il pubblico femminile delle scrittrici, abbastanza folto, è composto, prevalentemente, da donne che si sentono in qualche modo rappresentate da chi scrive [...]. Il pubblico femminile, sollecitato dai testi delle scrittrici, deve così fare i conti, da una parte, con ciò che delle donne si sa e si dice (ciò che gli uomini raccontano di loro), dall'altro con ciò che esse stesse vivono. Anche il pubblico femminile vive nel campo d'ambiguità: da una parte l'immaginario che la cultura maschile ha creato e che ha influito sulla mentalità anche delle donne e dall'altro la realtà delle cose, l'immediatezza del sentire. Le figure femminili, appartenenti all'immaginario creato dalla letteratura, sono un obbligato punto di riferimento anche per le scrittrici: con questo immaginario e con la propria esperienza, le scrittrici si scontrano e si incontrano, in questo immaginario collettivo devono collocare le loro storie, mediando con ciò che hanno l'urgenza di rappresentare. Dunque, lettrici e scrittrici fanno i conti con un «doppio»: da una parte ciò che del mondo si racconta (la visione, il punto di vista, $\mathrm{i}$ temi, la scala dei valori) e che ha inciso sul proprio stesso sentire, e dall'altra ciò che ciascuna di esse vive nella propria vita, nella

29. Cfr. Anna Santoro, «Narrativa di fine Ottocento: le scrittrici e il pubblico», cit., passim. 
propria esperienza sentimentale, artistica, intellettuale e politica. È la visione del mondo, è l'oggettività delle cose e dello sguardo, prima ancora dell'oggettività letterale, che in questo modo viene messa in discussione. ${ }^{30}$

Alcune di esse, infine, scrittrici squisite, come la Marchesa Colombi, Maria Messina, Lina Pietravalle, Sibilla Aleramo, e in altro modo Matilde Serao, Annie Vivanti, Eugenia Codronchi, Clelia Pellicano, trovano, sul piano della coscienza e del linguaggio, quella dimensione che io ho definito interna al "cammino dei desideri». "[...] le scrittrici, nel fare il loro linguaggio, hanno scoperto che se i desideri non vengono formalizzati con/nelle parole, essi non cambiano, le protagoniste non cambiano, le donne non cambiano. In tal caso, nel momento in cui si cerca di prendere la parola, ci si sente tagliate fuori, fuori posto, perché non si è trovato fino ad allora forza di camminare nei desideri, perché si è restate ferme al primo, mai soddisfatto»). ${ }^{31}$

In questo caso, la necessità del dire/dirsi, grazie a soluzioni narrative, scelte lessicali, metafore nuove, grazie al recupero del suono della voce e della capacità affabulativa orale, trova modo di dare forma alla percezione femminile, e di dire il non ancora pensato. Che però, vorrei attirare l'attenzione su questo punto, è già presente nel linguaggio femminile: nei gesti, nella voce, nella così detta intuizione, nei comportamenti.

Il linguaggio della parola nasce dal corpo. Nasce dall'incontro sempre nuovo tra corpo che percepisce, desidera, ha delle sensazioni, e necessità di dare forma al proprio desiderio, alla propria storia, alla propria curiosità, alla propria immaginazione. Il corpo c'è anche nel senso che tante tematiche femminili (o lette dallo sguardo femminile), provengono da un posizionamento, da un sapere, da un'attenzione, determinati proprio dal corpo femminile.

La interità propria della scrittura femminile realizzata dipende dalla interità percettiva delle donne, non nel senso di una unicità pacificata, ma nel senso che essa mette in campo mente, corpo, saperi, sentimento, affettività assieme al portato di ciascuno di questi elementi correlato agli altri. Al centro di questo processo c'è la scrittrice (la lettrice), che è corpo, linguaggio, capacità di selezionare e relazionare, e così via. Le parole sono corpose, nascono dalla voce, molte dalla voce di donna: è l'ordine del discorso (maschile) che ha sottratto la materialità delle parole, ne ha fatto un ordine astratto, tutto intellettuale. ${ }^{32}$ È l'ordine del discorso, fissato da regole maschili (la «maschilità» della gram-

30. Cfr. EAD., «Scrittrici», cit., passim.

31. Cfr. EAD., Il Novecento..., cit., p. 42-43.

32. Lavorando sulla lettura ad alta voce, mi è diventato chiaro come chi legge ad alta voce si fa tramite tra testo e ascoltatore: chi legge ad alta voce ha un rapporto di scambio con ciascuno dei due termini. Deve saper accogliere e rimandare il testo, valutando il contesto del testo e il contesto della platea d'ascolto, e tutto ciò possedendo in ogni caso un proprio contesto, una propria cultura che determina la sua lettura di tutti gli altri elementi. Chi legge per sé è tramite tra testo e se stessa /se stesso. Chi legge ad alta voce è scrittrice/lettrice e opera affinché chi ascolta sia lettrice/scrittrice. Chi legge ad alta voce, diviene a sua volta «testo». 
matica, per esempio), sono i canoni estetici e di giudizio, che attentano alla libertà creativa delle scrittrici e alla comprensione, da parte di chi legge, di dove sia tale libertà nei testi femminili.

In definitiva, imparai, e continuo ad imparare, che la differenza delle scrittrici impone la differenza della stessa lettrice, la quale, come le autrici che legge, non solo deve compiere un salto di prospettiva, uno spostamento di punto di vista e di posizionamento, idoneo a cogliere il nuovo della scrittura, ma deve assumere uno sguardo vergine, etico e creativo insieme.

Questa lettura mi è servita per verificare l'ipotesi da cui essa stessa nasceva: e cioè che la produzione letteraria femminile, in Italia, sin dai secoli passati, ha messo in crisi la nozione di Sistema Letterario. E mi ha aiutato (assieme alla riflessione sul lavoro di altre studiose) a segnare la fisionomia della buona lettrice $^{33}$ che, per essere tale, rivisita la letteratura e l'idea di letteratura, la lingua, gli scenari, i testi e i contesti. Solo con una lettura di parte, ${ }^{34}$ etica e creativa, è possibile interrogarsi sulle scritture delle donne e/o sul femminile nella letteratura, sulla «maschilità» della lingua italiana e sulla sua «inadeguatezza», ${ }^{35}$ e via di seguito.

La buona lettrice è eccentrica, eversiva, rispetto ai canoni tradizionali della ricerca scientifica. In questo senso è creativa: come la scrittrice legge e rappresenta l'incontro tra il proprio sguardo (ricco del proprio contesto) e lo sguardo della cosa guardata (il mondo), e, nel farlo, nel vivere la relazione, fa poesia, poiein, così la lettrice legge e rappresenta l'incontro tra il proprio sguardo e lo sguardo del testo, tesse la relazione e opera lo scarto rispetto ad una lettura convenzionale.

Leggere è guardare, è ascoltare, è accogliere ed elaborare la comunicazione (l'essere) dell'altra/o ed è anche comunicare a questa stessa comunicazione la propria lettura. Leggere è ricordare costantemente la parzialità della comunicazione stessa, dovuta sì al proprio posizionamento e punto di vista, ma anche all'inafferrabilità della complessità del testo (e del mondo) che si offre

33. Riguardo l'indagine sulla buona lettrice, mi permetto di rimandare ad altri miei lavori. Dopo il Catalogo, a parte i lavori di analisi applicata, sono tornata ad approfondire la questione a livello teorico. Cfr. per lo meno: Prefazione a Narratrici italiane dell'800, cit.; Prefazione alla seconda edizione del Catalogo... (dalle origini al 1900) (Napoli, 1990); «La lettura non è neutra», in Guida al Catalogo... (Napoli, 1990); «Ricerca e lettura delle scritture delle donne in Italia: Questioni di metodo», cit., p. 97-105; «Scrittura della differenza-Lettura della differenza. L'identità frammentata e la ricomposizione dei frammenti», in Daniela CoRONA (a cura di), Donne e scrittura, Palermo: La Luna, 1990; "Ricerca e lettura delle scritture delle donne in Italia: La lettrice», in Esperienze Letterarie, n.1, 1991, p. 99-106; "Il fatto è che ingrasso. Lettura di Un matrimonio in provincia della Marchesa Colombi», cit.; Introduzione a Il Novecento, cit.; "Scrittrici», cit.; Introduzione a Piccola Antologia di scrittrici campane, cit.; «Leggere, scrivere, linguaggi del corpo», cit.; «Leggere le scrittrici (italiane) del passato: questione di metodi. Impressioni e ricordi (1856-1864), Diario di Grazia Mancini Pierantoni», cit.

34. Cfr. Paola Bono, Esercizi di differenza-Letture partigiane del mondo e dei suoi testi, Genova: Costa \& Nolan, 1999; cfr. anche Leggendaria, E qui la critica?, maggio-giugno 1998.

35. Cfr. Patrizia VIOLI, L'infinito singolare, Verona: Essedue, 1986. 
esso stesso parziale a seconda dello sguardo che lo legge. Soggetto e oggetto si scambiano di ruolo continuamente: anzi, non c'è un soggetto e un oggetto, $\mathrm{ma}$, come ho scritto sopra, una relazione, un soggetto plurale. La buona lettrice sa che leggere un testo è l'incontro tra due soggetti che (per amore, interesse, curiosità, dovere...) si scambiano doni. Lei stessa è un testo che viene letto dal testo e che dal testo viene sollecitata a correlare, a mettere a fuoco, a richiamare alla memoria, eccetera. Dunque accoglie il testo e si lascia accogliere: si relaziona con esso. Sa che il testo si manifesta esso stesso altro a seconda dell'incontro che avviene con chi lo guarda, lo legge. Pensa che ciascuno dei termini (chi legge, ciò che viene letto), sia portatore di contesti, ricco, ambiguo. In questo senso nel leggere un testo la buona lettrice non lo affronta come macigno immutabile, corpo a tutto tondo da studiare e analizzare per comprendere ciò che possiede di per sé, perché quel «di per sé» in fondo è un'astrazione. Il testo è sempre in fieri, pur essendoci, tanto nel momento della stesura tanto nel momento della lettura. È in fieri, in trasformazione, la lettrice, ed è in fieri, in trasformazione, la scrittrice.

Su questa strada (la complessità di definire i contorni del testo e i contorni di chi legge, il testo non dato una volta per tutte, l'importanza di chi legge, e così via) ci si scontra a volte con la difficoltà di cogliere la soglia tra ciò che c'è nei nostri occhi e ciò che è fuori di essi (il testo, il testo-mondo). E questo, mi sembra, porta ad affrontare altre questioni importanti: c'è il testo senza lettrice/lettore? Lo sguardo fa il testo? Guardare è già interferire?

A queste domande cerco di rispondere con grande cautela: nel momento stesso in cui affermo la necessità della libertà della lettura (che rompa anche essa canoni, schemi e rituali), nel momento in cui mi interrogo su cosa sia il senso letterale e dubito di cosa e dove sia la realtà, cerco però di tenere ben presente che esiste, in piena dignità, l'altra/o (in questo caso, il testo), e che le motivazioni per cui si legge (il testo o il mondo) fissano in qualche modo non solo il punto di vista, ma la prospettiva, l'oggetto, che in questo modo risulta reale e con il quale bisogna fare $i$ conti. In realtà, ritengo che $i$ «limiti dell'interpretazione» (gli eccessi dell'interpretazione) dipendano anche da una certa superfetazione dell'ego (maschile) che si pone come punto di riferimento assoluto e continuo, il che limita la sua stessa capacità di percezione e gli impedisce di leggere il diverso/la diversa.

Il testo recupera un posto privilegiato invece proprio grazie alla capacità creativa della lettrice. E alla capacità etica: il riconoscimento dell'esistenza del testo nasce dal rispetto dell'altra/o, dalla coscienza della parzialità della propria lettura, e dalla valutazione che, quanto più un testo è un mondo (cioè articolato, ricco), tanto più può e deve essere accolto ed elaborato, lasciando che ci accolga e ci elabori. Il che è proprio del femminile. L'accoglienza a cui accennavo prima è elaborazione, cioè trasformazione dell'oggetto elaborato e del soggetto elaborante. Nel leggere noi donne ci sentiamo sempre implicate: per questo ci commuoviamo, ci muoviamo con, cioè relazioniamo ciò che leggiamo con noi stesse, ma non riduciamo il tutto a noi: anzi, ci «mettiamo in discussione» come si diceva una volta. Leggiamo noi e siamo lette dal noi che, 
grazie al rapporto della lettura del sé, legge fuori, il testo. Nello stesso tempo è il fuori, il testo, che permette la lettura del sé. Nel leggere un testo (il mondo), la buona lettrice modifica se stessa perché ascolta (legge) le modalità della sua stessa ricezione. La lettura ci cambia. Come ci cambia la scrittura. La scrittura, come la lettura è, anche, un segno di confidenza, di affidamento: alle parole per esempio. Le scrittrici che sono riuscite a dare forma alla propria lettura, cioè a rendere reale ciò che nasce dall'incontro del proprio sguardo con lo sguardo della cosa guardata, hanno fatto/fanno poesia. In certo senso hanno lasciato che la poesia che c'è, a saperla vedere, nel mondo, potesse prendere forma grazie al proprio tramite. Allo stesso modo la lettura (come la scrittura) ci arricchisce proprio se e quando ci dimentichiamo di noi stesse, pur essendo molto presenti.

Anche questo autorizza a riferirsi alle scritture critiche delle donne come a scritture creative. Creative di un genere fuori dai canoni. E questo ci autorizza anche a sottolineare l'eticità della lettura di genere. La buona lettrice, infatti, continua a pensare che esista la passione della scrittura, e la scrittura che le interessa è quella che riesce a dare forma al desiderio. Così si relaziona, per desiderio, al desiderio dell'altra/dell'altro, e, nel leggere i segni, è (deve essere) in grado di risalire da essi alle citazioni, di comprendere le metafore e il non detto, che in realtà è il detto in altro modo. La buona lettrice svela le nuove metafore delle scrittrici perché è in grado di coglierle. Il lavoro di riappropriazione delle parole e del discorso è il lavoro che oggi tocca non solo alla scrittrice, ma anche alla buona lettrice.

Il punto di vista di chi legge non è dunque una categoria mentale, una semplice (nel senso di: unica, staccata dal corpo, dalla percezione...) capacità delle donne di intellettualizzare i propri saperi, ma è coinvolgimento della propria interità e unicità nell'atto della lettura.

Il corpo nella scrittura è tramite tra percezione e formalizzazione. È un modello comunicativo. Creatore di segni. Le due unità/unicità (della scrittrice e della lettrice) si incontrano nei loro sguardi fatti di intelligenza, di sapere, di sensibilità, di sensazioni, di citazioni di odori, sapori, evocazioni tattili, rossori, eccetera. Le scritture-letture delle donne sono punto di incontro e unità tra tradizione orale (il parlare tra donne, l'affabulazione, la voce) e discorso letterario.

Il femminile nella letteratura sta, dunque, nelle forme che le scrittrici hanno dato e danno alla propria immaginazione, al proprio desiderio, al proprio sapere, al proprio punto di vista, (grazie) alla propria scrittura. Nelle scritture delle donne ci sono segni di inedite e straordinarie soluzioni e invenzioni che bisogna imparare a leggere, non solo ai fini del recupero della memoria necessaria a noi donne del xx secolo (ora del Terzo Millennio), ma perché esemplari di capacità (diverse dalle convenzionali) ${ }^{36}$ di accoglienza e di elaborazione di un

36. EAD.: «Sarebbe mille volte un peccato se le donne scrivessero come gli uomini o vivessero come gli uomini o assumessero l'aspetto di uomini, perché se due sessi sono insufficienti, considerata la vastità e varietà del mondo, come faremmo mai con uno solo ?» (p. 179-180); cfr. anche: Marie Luise WANDRUSZKA, Scrivere il mondo, Torino: Rosemberg e Sellier, 1996. 
linguaggio, di una poetica, tutti inevitabilmente interni, con diversi esiti, a quello che ho definito "campo di ambiguità».

[...] Il campo d'ambiguità è costituito da questo spazio dove il sistema (della scrittura o dei comportamenti, maschile) della tradizione e della omologazione, sistema letterario canonizzato, si incrocia nelle donne con un sistema altro (della scrittura o dei comportamenti, femminile) non stabile, né definito da astrazioni o da un ordine compatto, appunto perché questa compattezza, questo "campo chiuso", proprio di ogni sistema, non gli appartiene. Perché è altro, segnato dall'accoglienza e dall'articolazione. ${ }^{37}$

Tra la tendenza, il fascino (e, anche, l'opportunità) della omologazione e della normalizzazione da una parte e la necessità del dirsi, del dare forma al sé, al proprio sguardo, ai propri interessi, le scrittrici (ciascuna a suo modo) hanno cercato e creato un linguaggio più o meno innovativo, più o meno ossequiente dei canoni, un linguaggio di per sé eversivo, che è riuscito a dare forma alla necessità/ricerca del dire altro e del dirlo in altro modo.

[...] La soggettività femminile nella scrittura (che porta innovazioni forti nella tradizione) si esplica a vari livelli: con il mettere al centro, introducendola come protagonista, una donna, con il disegnare uno scenario dove la relazione tra donne, sia pure diverse, crea un clima, un'atmosfera e permette una grammatica che rompe lo schema in $o$ e in $i$ aprendo il suono della pagina in $a$ e $e$ riappropriandosi della parola detta (cioè del suono della parola e della voce), che è parte del linguaggio del corpo. Ancora, la soggettività femminile si esplica con l'affrontare delle tematiche "trasparenti» per lo sguardo dello scrittore (e del lettore), dando visibilità non solo ad esse ma ad un punto di vista inedito e cosciente di sé che dunque abbraccia la visione del mondo: è da qui che nascono i grandi libri di denuncia della propria condizione, dei comportamenti maschili, dei conflitti tra i due generi, ma anche dei guasti per tutti (es. la guerra, la violenza...) e anche da qui nascono i grandi libri di felicità e di gioco, di libertà. Va anche aggiunto che la capacità affabulativa femminile, soprattutto per il passato, non si poggia sulla "meravigliosità» delle avventure («l'esperienza memorabile» dei viaggiatori, dei cacciatori, dei guerrieri) ma sulla «meravigliosità» della immaginazione che pone al centro il rapporto individuo-mondo. In questo modo le scrittrici, attraverso la fantasia e il sogno, legato al quotidiano, il quotidiano trasfigurano, a volte per allontanarsene ma spesso per tornarci sopra, forti di una immaginazione, di un desiderio che possa trasformarlo. Questo è il nucleo forte della soggettività femminile nella scrittura. $[\ldots] .^{38}$

37. Cfr. Anna Santoro, Il Novecento. Antologia..., cit., p. 23. Cfr. anche: EAD., «Ricerca e lettura...1) e 2)», cit.; EAD., "Scrittrici», cit.

38. Cfr. Anna Santoro, Scrittrici, cit. 